\title{
Nachweis von Chloridspuren in der Gelatine.
}

Von Dr. Lüp po-Cramer.

(Wissenschaftliches Laboratorium der Dr. C. SchleuBner-Aktiengesellschaft in Frankfurt a. M.)

Die Gelatinesorten des Handels, auch die für photographische $Z$ wecke u. a. mit besonderer Sorgfalt hergestellten Marken, enthalten von ihrer Herstellung her fast stets mehr oder weniger grobe Mengen von Chlorid in Form von $\mathrm{CaCl}_{2}$. Für die Herstellung der gewöhnlichen photographischen Präparate und die meisten andern Zwecke werden kleine Mengen von Chlorsalzen zwar wohl kaum schaden, doch können sie für wissenschaftliche Versuche, z. B. auch zur Herstellung von Lippmann-Emulsionen, bei denen man möglichst mit genau äquivalenten Mengen von Silbernitrat und Haloidsalz arbeitet, immerhin von Bedeutung sein.

Sucht man nun einen Chloridgehalt der Gelatine durch Zusatz von Silberlösung zur Gelatinelösung nachzuweisen, so versagt diese Probe, sobald es sich um kleine Mengen von Chloriden handelt. Es bildet sich in dem starken Schutzkolloid eben kolloides Chlorsilber in so auBerordentlich feiner Solform, daB keinerlei sichtbare Trübung auftritt.

Auf eine sehr einfache Weise kann man aber die geringsten Spuren von Chlor in der Gelatine nachweisen, indem man nämlich etwas Gelatinelösung (10 Proz.) auf einer Glasplatte erstarren läBt und dann Tropfen von Silbernitrat (10 Proz.) auf die Platte bringt, in derselben Weise also, wie es R. Ed. Liesegang in seinen grundlegenden Untersuchungen: "Reaktionen in Gallerten " 1) beschrieben hat. Der Silbertropfen bewirkt bei Anwesenheit von geringen Chloridmengen in der Gelatine zunächst keinerlei Trübung. Nach mehr oder weniger langer Zeit bildet sich jedoch auBer$\mathrm{h}$ alb des Tropfens ein zuerst opalisierender und nach und nach immer trüber werdender Kreis, der in einigen Stunden je nach der Größe des Tropfens und dem Chloridgehalt der Gelatine einen Durchmesser bis zu 1 und $2 \mathrm{~cm}$ erreicht. Noch bei einem Chlornatriumgehalt von 0,001 Proz., bezogen auf die trockene Gelatine, werden die Chlorsilberkreise außerhalb des Tropfens ganz opak weiß. Durch Vergleich einer gewöhnlichen Gelatine mit einer durch gründliches Auswaschen gereinigten, der man steigende Mengen von Chlorid zusetzt, kann

1) R. Ed. Liesegang, Reaktionen in Gallerten (Dusseldort 1898). man sich leicht ein Bild davon machen, wie auberordentlich fein jene Gallertreaktion ist.

Es war nun von besonderem Interesse, festzustellen, warum die Gelatine unterhalb des Silbertropfens ungetrübt bleibt, während sich anscheinend nur in dem Kreise außerhalb des Tropfens Chlorsilber bildet. Die Lösung dieser Frage ergibt sich, wenn man die Gallertplatten nach stattgehabter Diffusion der Silberlösung dem Tageslichte aussetzt. Die weiben Ringe werden dann nach und nach graublau, die farblosen Stellen unter dem Silbertropfen rotgelb, was in Analogie mit andern photographischen Erscheinungen beweist, dab unter dem Tropfen zwar auch Chlorsilber sich befindet, dab dieses aber in auBerordentlich viel feiner verteilter Form vorhanden ist. Nun hat Lie segang a. a. O. gezeigt, dak bei jenen Gallertreaktionen die Salze wandern. Andererseits konstatierte der Verfasser ${ }^{2}$ ) bei seinen Untersuchungen über die Reifung photographischer Emulsionen, daB das Korn der Silberhaloide zwar nicht durch überschüssiges Silbernitrat, wohl aber durch Haloidsalze "gereift", d. h. vergröbert wird. Die Fragestellung bei dem beschriebenen Phänomen ist also nicht: „Warum bleibt die Stelle unter dem Tropfen klar?", sondern das zunächst Auffällige liegt darin, dab das innerhalb der Gallerte sich bildende kolloide Chlorsilber nur a $\mathrm{B}$ erhalb des Tropfens eine so rasche Reifung erfährt. Hier wandert eben von auBen sukzessive Chlorid nach, das das ursprünglich kolloide $\mathrm{AgCl}$ reift, während unter dem Silbernitrattropfen hierzu keine Gelegenheit ist, da hier stets Silbersalz im UeberschuB bleibt.

Die von Liesegang beschriebene Wanderung des Chlorides nach dem Silbertropfen zu zeigte sich auch bei meinen Versuchen recht deutlich. Zwischen je zwei Silbertropfen bildet sich nämlich stets ein vollständig chlorfreier oH of, der beim Anlaufen des Chlorsilbers im Lichte völlig ungefärbt blieb.

Die Methode, chemische Reaktionen innerhalb einer erstarrten Gallerte anzustellen, ist einer sehr allgemeinen Anwendung fähig und besonders für das Studium des photographischen

2) Lüppo-Cramer, Photogr. Probleme (Halle 1907), S. 23. 
Reifungsprozesses ${ }^{3}$ ) sehr fruchtbar. Die oben beim Chlorsilber beschriebene Reifung durch das nachdiffundierende Salz zeigt sich u. a. auch sehr instruktiv bei der Bildung von Quecksilberjodid innerhalb der Gelatine. Setzt man Tropfen einer vierprozentigen Sublimat-

3) Vgl. auch Lii ppo-Cramer, Photogr. Korresp. 1906 , S. 485. lösung auf eine Gallerte, die 0,01 Proz. Jodkalium enthält, so bilden sich zuerst Ringe von gelblichwe i B em Quecksilberjodid, das mit zunehmender Reifung in die rote Form übergeht, wie ich dies bei Emulsionen schon vor Jahren ${ }^{4}$ ) beschrieb.

4) Lü p p o-Cramer, E der's Jahrb. f. Phot. 1903, S. 30; Photogr. Korresp. 1903, S. 719.

\section{Ueber Kapillar- und Adsorptionsanalyse.}

Auszug aus meinen seit 1861 bis 1907 erschienenen Publikationen iber das auf Kapillaritats - und (Fortsetzung) Adsorptionserscheinungen beruhende Gebiet der Kapillaranal ys e.

Kapitel V. Anwendung der Kapillaranalyse in der physiologischen Chemie.

2. Kapillaranalytische Untersuchungen des Harns.

Nachdem ich seit Anfang der 80er Jahre den Harn in das Bereich meiner kapillaranalytischen Untersuchungen gezogen hatte (siehe Hefte des k. k. Technologischen Gewerbemuseums in Wien $1887 / 88$ ), wurde es mir während der Monate November und Dezember 1902 infolge freundlichen, hier wieder bestens verdankten Entgegenkommens des damaligen Oberarztes der Klinik für innere Medizin im Bürgerspital zu Basel, des Herrn Prof. Dr. W. $\mathrm{H}$ is, und seines damaligen ersten Assistenten, Herrn Prof. Dr. Rud. S t a e helin, ermöglicht, eine systematische kapillaranalytische Untersuchungsreihe von 507 frischen Harnproben von $178 \mathrm{Kranken}$ in $86 \mathrm{Krankheitsfällen} \mathrm{vorzunehmen.}$ Ich verweise auf meine Publikation: "Studien über die Anwendung der Kapillaranalyse I bei Harnuntersuchungen, II bei vitalen Tinktionsversuchen ", Verhandlungen derNaturforsch. Ges, in Basel, Bd. XVII, 1904. (Text Seiten 1 bis 198, Tafeln 1 bis 119.) Es wäre mir natürlich sehr erwünscht gewesen, jeden Harn einer genauen physikalischen und chemischen und die in ihm suspendierten Ausscheidungen einer mikroskopischen und chemischen Prüfung zu unterziehen, was für die Vergleichung mit den Resultaten der Kapillaranalyse sehr wichtig wäre. Leider feblte mir dazu Zeit und Mithilfe.

Was die Farbe des Harns anbetrifft, so ist sie beim normalen Harne bekanntlich mehr oder weniger hell bis lebhaft gelb, beim konzentrierteren bis rotgelb und sogar rotbraun. Aber selbst bei starkem Gehalte an festen Stoffen, also bei hohem spezifischen Gewichte, kann der Harn, z. B. diabetischer, hellgelb sein. Stark gelb- bis braunrote Farbe deutet auf
Urobilin, fleischwasserrote bis rubinrote auf wenig bis viel Blutfarbstoff, braungelbe bis grünliche und gelber Schaum beim Schütteln auf Gallenfarbstoff (ikterischer Harn), tiefbraune Farbe auf indoxylschwefelsaure Salze (Indikanurie).

Von organisierten Sedimenten, ohne jedoch in nähere mikroskopische Prüfung eingetreten zu sein, glaube ich verschiedene auf Eintauchszonen beobachtet zu haben, namentlich wenn der Harn vorher durcheinander gerührt worden war.

Von amorphen Sedimenten ist das Ammoniakurat, welches feine, teils einzelne, teils in Gruppen beisammenliegende Körnchen oder dunkle an ihrer Peripherie mit radienförmig stehenden Kristallnadeln versehene Kugeln bildet und in Essigsäure löslich ist, zu nennen. Ich glaube es in der obersten Endzone von Kapillarstreifen beobachtet zu haben; mit der Zeit aber verschwindet es infolge Fäulnisprozesses, so daß nun an der Stelle, wo sich die zahlreichen Kügelchen befanden, der leere weiße Striemen des Filtrierpapiers inmitten der gelblichen bis gelben und bräunlichgelben Urochromfärbung zu sehen ist. Ferner beobachtete ich hier und da die weißen in Essigsäure ohne Gasentwicklung löslichen Körnchen von basischem Kalk - und Magnesiaphosphat. In Essigsäure mit Gasentwicklung lösliche Körnchen bestehen aus $K a r b o n a t e n d e r s e l b e n a l k a l i s c h e n$ Erden. Hantelförmige, in Essigsäure ebenfalls unter Gasentwicklung lösliche Massen bestehen aus Kalziumkarbonat. Auch in amorphen hantelförmigen, in Ammoniak und in konzentrierter Salzsäure unlöslichen Massen zeigt sich der $\mathrm{sch}$ wefelsaure Kalk, ebenso der oxalsaure Kalk, welcher auch unlöslich in Essigsäure, aber löslich in konzentrierter Salz- 